\section{FLT3-Hemmer bei systemischer Mastozytose}

Bei seltenen Neoplasien muss manchmal auf historische Daten zurückgegriffen werden, um die Wirksamkeit und Sicherheit einer potenziellen Therapie zu untersuchen. In zwei einarmigen Phase-II-Studien hatte sich bei Einsatz des auf FLT3 und D816V-mutierte KIT zielende Midostaurin bei Patienten mit einer aggressiven systemischen Mastozytose ein gutes oder zumindest partielles Ansprechen von erkrankungsbedingten Organschäden gezeigt. Andreas Reiter, Mannheim, berichtete jetzt von dem Vergleich von 89 Patienten aus diesen Studien mit Daten von 42 Patienten aus einem deutschen Register aus demselben Zeitraum, die nicht mit Midostaurin behandelt worden waren [EHA. 2017;Abstr S788]. Midostaurin halbierte etwa das Mortalitätsrisiko, das mediane Gesamtüberleben lag in den Phase-IIStudien gepoolt bei 41,4 Monaten, im Register dagegen bei 19,5 Monaten. In einer Matched-pair-Analyse fiel der Vorteil etwas geringer aus (27,8 vs. 19,5 Monate). Auch in einer multivariaten Analyse blieb der Vorteil für Midostaurin bestehen. Eine weitere Studie mit historischer Kontrolle kam zu ähnlichen Ergebnissen [Chandesris $\mathrm{MO}$ et al. N Engl J Med. 2016;374(26):2605-7]. Dass solche Studien nicht optimal sind, weiß Reiter auch, aber betonte: „Wir haben einfach nichts Besseres." Friederike Klein

Bericht vom 22. Kongress der European Hematology Association vom 22. bis 25. Juni 2017 in Madrid, Spanien siko für die Entwicklung venöser Thromboembolien (VTE), erinnerte Alejandro Lazo-Langner, London, Kanada. Dieses Risiko kann durch Antikoagulanzien effektiv gesenkt werden. Allerdings steigern diese Substanzen auch die Gefahr von Blutungskomplikationen, die bei Tumorpatienten ohnehin erhöht ist. Bislang ging man davon aus, dass Rezidivthrombosen das Sterberisiko in vergleichbarem Maße erhöhen wie durch die Antikoagulation bedingte Blutungsepisoden. Doch ist unklar, ob dies auch für Krebspatienten gilt.

Im Rahmen einer retrospektiven populationsbasierten Beobachtungsstudie untersuchte die Arbeitsgruppe um LazoLangner daher, ob ältere Tumorpatienten (> 65 Jahre) stärker gefährdet sind, an einem VTE-Rezidiv oder an einer Blutung aufgrund der Antikoagulation zu sterben [Lazo-Langner A et al. EHA. 2017;Abstr S441]. Sie werteten die Daten von fast 7.000 VTE bei im Schnitt 75-jährigen Krebspatienten aus. Die meisten (75\%) waren mit niedermolekularem Heparin mit oder ohne Warfarin antikoaguliert worden. $22 \%$ der Patienten hatten nur Warfarin, $3 \%$ Rivaroxaban erhalten.

Rezidivierende VTE waren erheblich häufiger als Blutungskomplikationen: Sechs Monate nach der Index-VTE waren 235 schwere gastrointestinale und intrakranielle Blutungen (3\%), aber 1.185 Rezidivthrombosen (17\%) aufge- treten. Die blutungsbedingte Mortalitätsrate nach sieben Tagen war jedoch mit $11 \%$ erheblich höher als die VTEbedingte Sterblichkeit mit nur 0,5\%. Das Risiko, an einer Blutung zu sterben, sei damit um ein Mehrfaches höher als bei einem VTE-Rezidiv, schlussfolgerte Lazo-Langner. Unterschiede zwischen den verschiedenen Antikoagulanzien wurden nicht festgestellt. Das Ergebnis der retrospektiven Untersuchung sollte in weiteren Studien verifiziert werden, forderte Lazo-Langner. Auch sollte das hohe Blutungsrisiko vor Initiierung einer Antikoagulation bei älteren Krebspatienten berücksichtigt werden.

\section{Myelom: höhere Effektivität von Zweitgenerations-PI}

In der Phase-III-Studie ENDEAVOR erhielten mehr als 920 Patienten mit rezidiviertem oder refraktärem multiplem Myelom (MM) randomisiert entweder die Standardtherapie mit Bortezomib plus Dexamethason (Vd) oder den Zweitgenerations-Proteasominhibitor (PI) Carfilzomib plus Dexamethason (Kd). Nach einem Follow-up von median 11,2 Monaten hatte Kd im Vergleich zu Vd zu einem doppelt so langen PFS (primärer Endpunkt) geführt (18,7 vs. 9,4 Monate; HR 0,53; $\mathrm{p}<0,0001$ ) [Dimopolulos MA et al. Lancet Oncol. 2016;17(1):27-38].

Jetzt liegt eine Update von ENDEAVOR vor, das erstmals Ergebnisse der vorab geplanten Interimsanalyse zum Gesamtüberleben (OS) liefert [Dimopoulos MA et al. EHA. 2017;Abstr S458]. Die Nachbeobachtung erstreckt sich über rund 37 Monate; ausgewertet wurden $79 \%$ der erwarteten Ereignisse.

\section{Auch Gesamtüberleben länger}

Auch hinsichtlich des OS erwies sich das Kd-Regime im Vergleich zum bisherigen Standard als überlegen (median 47,6 vs. 40 Monate; HR 0,791; p = 0,0100). Das KdRegime war in allen untersuchten Subgruppen vorteilhaft. Meletios Dimopoulos, Athen, Griechenland, wertete die Verlängerung des PFS und OS durch Carfilzomib als „klinisch relevant“.

In puncto Verträglichkeit gab es auch nach längerem Follow-up keine unerwarteten Sicherheitssignale: Die Rate an Nebenwirkungen vom Grad $\geq 3$ war im Carfilzomib-Arm um rund $10 \%$ höher als im Bortezomib-Arm (81,4 vs. 71,1\%). Dabei sei jedoch zu berücksichtigen, so betonte Dimopoulos, dass Patienten im Kd-Arm gut 21 Wochen länger behandelt worden seien als die im Vd-Arm. Adjustiert man für die unterschiedliche Therapiedauer, so waren die Raten an Nebenwirkungen vom Grad $\geq 3$ ebenso wie die schwerwiegender Nebenwirkungen zwischen beiden Studiengruppen vergleichbar. Nebenwirkungsbedingte Therapieabbrüche traten bei einem Viertel der mit Kd und 21,7\% der mit Vd behandelten Patienten auf.

Hypertonie und Herzinsuffizienz (beide Grad $\geq 3$ ) waren unter Carfilzomib häufiger als mit dem Erstgenerations-PI (15,6 vs. $3,3 \%$ bzw. 5,8 vs. $2,0 \%$ ). Periphere Neuropathien (PN) aller Schweregrade waren unter dem Vd-Regime mehr als doppelt so häufig wie unter Carfilzomib (54,6 vs. $21 \%$ ). PN vom Grad $\geq 3$ wurden bei 9,6\% der mit Bortezomib, aber nur 2,4\% der mit Carfilzomib behandelten Patienten verzeichnet.

\section{CD19-Antikörper beim diffus- großzelligen Lymphom}

Bei Patienten mit rezidiviertem oder refraktärem diffus-großzelligem B-ZellLymphom (DLBCL) hat sich der CD19. Antikörper MOR208 in Kombination mit Lenalidomid bei guter Verträglichkeit als sehr effektiv erwiesen [Maddocks KJ et al. EHA. 2017;Abstr S470]. Das Oberflä- 